\title{
Magnitude of fixation effect as influenced by estrogen fluctuations during the menstrual cycle
}

\author{
VICKI L. FLAHERTY and DEBRA COWART-STECKLER \\ Mary Washington College, Fredericksburg, Virginia \\ and \\ ROBERT H. POLLACK \\ University of Georgia, Athens, Georgia
}

\begin{abstract}
The relationship between fluctuations in estrogen level and magnitude of fixation effect was investigated using 14 normally menstruating females as the experimental group and 14 oral contraceptive users as the control. The experimental group was expected to show the greater magnitude of fixation effect. Overall, the magnitude of fixation effect was expected to be greater at the high estrogen level. The difference between the groups was expected to be greater at the high estrogen level than at the low. Stimuli were circles presented with a two-channel tachistoscope. Magnitude of fixation effect was calculated by subtracting the experimental trial point of subjective equality (PSE) from the baseline PSE. Data were analyzed using a one-between/one-within repeated measures design. The results generally confirmed our hypotheses and suggested that high estrogen levels enhance the magnitude of fixation effect. The findings support earlier speculations that male/female differences in magnitude of fixation effect might be due to female hormonal fluctuations.
\end{abstract}

Fixation effects are perceived changes in a stimulus figure that occur during and after prolonged inspection of that figure, and are based on the phenomenon of figural aftereffect (FAE) of size (Kohler \& Wallach, 1944). The conventional effect is a perceived change in the size of a test figure (TF) that is presented after prolonged viewing of an inspection figure (IF).

Cowart-Steckler and Pollack (1982) found that with both circular and triangular figures there was a greater magnitude of fixation effect for female subjects than for male subjects. Other researchers have found similar results (Kline \& Nestor, 1977; Kline \& Orme-Rogers, 1978). Kline and Nestor (1977) suggested that these findings may be related to cyclical hormone changes in young females. Diamond, Diamond, and Mast (1972) showed that visual threshold reductions were related to cyclical hormone changes in young females, and such changes may be related to the persistence of visual stimuli (Kline \& Nestor, 1977). Stimulus persistence is important in the fixation effect because the persistence of the IF after it has been removed may be a cause for the perceived changes in the TF (Cowart-Steckler \& Pollack, 1982).

Studies showing male/female differences in the magnitude of fixation effect have often claimed that female hormonal fluctuations are responsible for these differences, but research has failed to address this hypothesis to date. Thus, the point of the present study was to deter-

Address correspondence to R. H. Pollack, Department of Psychology, University of Georgia, Athens, GA 30602. mine whether female hormonal fluctuation is a plausible explanation for the observed sex differences in the magnitude of fixation effect. The relationship between hormonal variations and the magnitude of fixation effect in females was investigated using normally menstruating females as the experimental group and oral contraceptive users as the control group. We expected a difference between the two groups, with normally menstruating females showing the greater magnitude of fixation effect.

Estrogen level, as determined by menstrual cycle fluctuations, was used as the independent variable. Because recent research has shown that at high hormone levels, females' sensory sensitivity is greater (Barris, Dawson, \& Theis, 1980; Scher, Pionk, \& Purcell, 1981; Shaffer \& Payne, 1982), we predicted that magnitude of fixation effect would be greater at high estrogen levels for both groups.

In addition, the difference between the groups was expected to be greater at the high estrogen level than at the low estrogen level. Estrogen level should be relatively low for both groups during menstruation; however, halfway through the menstrual cycle, estrogen level should be higher for normally menstruating females, because the pill actually works to keep estrogen level moderately low (so that ovulation will not occur) in oral contraceptive users.

\section{METHOD}

\section{Subjects}

Twenty-eight female undergraduate students served as subjects. The subjects were either volunteers or participants in the psychology depart- 
ment's research pool. Fourteen subjects had regular menstrual cycles and served as the experimental group; 14 were oral contraceptive users and served as the control group. Only subjects using the "combination type" pill were inciuded in the control group.

All subjects had 20/30 corrected or uncorrected vision as determined by the Bausch and Lomb Modified Orthorator. Subjects whose vision was corrected with glasses were not included because the tachistoscope would not accommodate frames.

\section{Materials}

A Bausch and Lomb Modified Orthorator was used to test subject's vision.

A Scientific Prototype two-channel tachistoscope, Model N-900, was used to present stimuli. The maximum horizontal visual angle subtended $2.9^{\circ}$ and the maximum vertical angle was $0^{\circ} 44^{\prime}$. Reflected illumination was 6.5 apparent footcandles as measured by the Macbeth Illuminometer.

The stimuli were circles drawn on standard white index cards with black India ink. The circle outlines were $.5 \mathrm{~mm}$ thick. The IF consisted of 1 circle $19 \mathrm{~mm}$ in diameter. The TF consisted of 2 circles, a standard circle identical to the IF circle and one of 14 comparison circles. The comparison circles varied in size from 12 to $15 \mathrm{~mm}$ in diameter, increasing in increments of $1 \mathrm{~mm}$ (see Figure 1).

\section{Procedure}

The experimenter informed the subjects of the importance of accurately scheduling experimental sessions. Experimental sessions were scheduled when the subject's estrogen level was low and when it was high. Estrogen level for both the control and experimental groups was considered low on the 2, 3, or 4 day after the menstrual cycle began, and estrogen level was considered high on the 12,13 , or 14 day after the onset of the menstruation (Masters, Johnson, \& Kolodney, 1985). The beginning of the menstrual cycle was defined as the 1 day of menstrual flow (see Figure 2).

To control for factors such as fatigue and circadian rhythm, both experimental sessions took place at approximately the same time of day. The second session, which occurred approximately 10 days after the first session, was scheduled no later than $1 \mathrm{~h}$ after the time of the first session and no earlier than $1 \mathrm{~h}$ before the time of the first session. To counterbalance for possible first-time effects, for half of the subjects the first experimental session took place when the subject's estrogen level was low, and for half of the subjects the first session tock place when the subject's estrogen level was high.

The experimental session was conducted in a sound-attenuated cubicle. Except for a small light used by the experimenter, the cubicle was kept dark so the subject could make size judgments as accurately as possible. The subject was first instructed to fixate on the red dot that appeared in the center of the tachistoscope's visual field. The trials were explained to the subject, and the subject was asked to indicate after each exposure which of the two TF circles appeared larger, the one on the right or the one on the left.
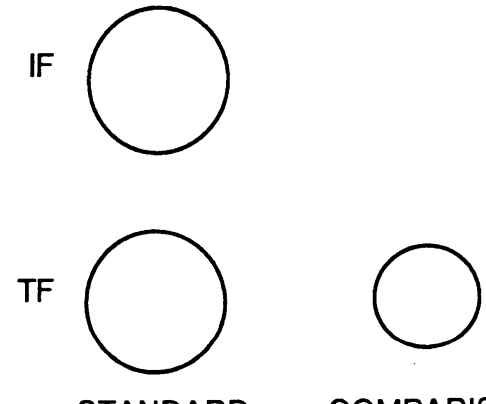

STANDARD

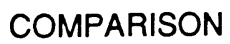

Figure 1. The stimuli: the inspection figure (IF) and a test figure (TF).

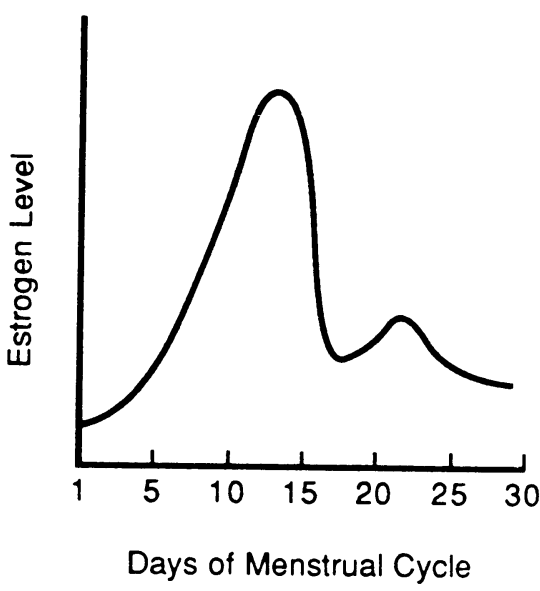

Figure 2. Estrogen level over the menstrual cycle.

The experimental sessions consisted of 18 trials. On half of the trials the IF and standard appeared on the right and on half they appeared on the left. Initial presentations of the IF and standard on the right and on the left were counterbalanced.

To obtain baseline data, the experimenter had each subject view two trials of TFs before and after five experimental trials with the IF and standard on the right and before and after five experimental trials with the IF and standard on the left. During these baseline trials TFs were presented for $500 \mathrm{msec}$. The stimuli in these baseline trials, as well as in the experimental trials, were presented according to Piaget's (1961/1969) converging method of limits.

During experimental trials the IF was presented for $9.9 \mathrm{sec}$, followed by the TF, which was presented for $500 \mathrm{msec}$. There was no interstimulus interval between the IF and TF presentations. During the experimental trials, there was a 5-sec interval between exposures. This interval was used so that subsequent judgments would not be influenced by previous exposures.

\section{RESULTS}

To determine the magnitude of fixation effect, we calculated a difference value for each subject at both high and low estrogen levels by subtracting point of subjective equality (PSE) during experimental trials from PSE at baseline. The data were analyzed with a onebetween/one-within repeated measures design, using the difference values. For descriptive statistics see Table 1 .

A significant main effect for groups was obtained $[F(1,26)=10.64, p<.0031]$, with regularly menstruating females showing a greater magnitude of fixation effect. A significant main effect for estrogen level was also obtained $[F(1,26)=7.81, p<.0096]$, with the greater magnitude of fixation effect occurring at the high estrogen level. The interaction was not significant.

\section{DISCUSSION}

As we hypothesized, normally menstruating females did show a greater magnitude of fixation effect than oral contraceptive users, and overall the magnitude of fixation effect was greater at the high estrogen level than at the low estrogen level. Counter to our expectation, however, the difference between the groups was smaller at the high estrogen level than at the low estrogen level. If we had used a more precise method 
Table 1

Mean Point of Subjective Equality

\begin{tabular}{lll} 
& \multicolumn{2}{c}{ Estrogen Level } \\
\cline { 2 - 3 } Group & High & Low \\
\hline Control & .3955 & .1589 \\
Experimental & .5357 & .4670 \\
\hline
\end{tabular}

Note-Larger values represent greater magnitude of fixation effect.

of measuring estrogen level, such as taking basal metabolic temperature, the results might have been in the predicted direction.

Generally, the findings are supportive of earlier suggestions that male/female differences in magnitude of fixation effect may be due to female hormonal fluctuations (Kline \& Nestor, 1977). The results suggest that higher estrogen levels, resulting from fluctuations during the normal menstrual cycle, enhance the magnitude of fixation effect. This is in line with other research on visual sensitivity and menstrual cycle fluctuations, which shows that visual thresholds tend to be lower at the time of ovulation (Parlee, 1983).

\section{REFERENCES}

Barris, M. C., Dawson, W. W., \& Theis, C. L. (1980). The visual sensitivity of women during the menstrual cycle. Documenta Ophthalmologica, 49, 293-301.

Cowart-Steckler, D., \& Pollack, R. H. (1982). The aftereffects of prolonged perception of shape. Bulletin of the Psychonomic Society, 20, 239-241.
Diamond, M., Diamond, A. L., \& Mast, M. (1972). Visual sensitivity and sexual arousal levels during the menstrual cycle. Journal of Nervous \& Mental Diseases, 155, 170-176.

Kline, D. W., \& Nestor, S. (1977). Persistence of complementary afterimages as a function of adult age and exposure duration. $E x$ perimental Aging Research, 3, 191-201.

Kline, D. W., \& Orme-Rogers, C. (1978). Examination of stimulus persistence as the basis for superior visual identification performance among older adults. Journal of Gerontology, 33, 76-81.

KoHLER, W., \& Wallach, H. (1944). Figural aftereffects: An investigation of visual processes. Proceedings of the American Philosophical Society, 88, 269-357.

Masters, W. H., Johnson, V. E., \& Kolodney, R. C. (1985). Human sexuality (2nd ed.). Boston: Little, Brown.

Parlee, M. B. (1983). Menstrual rhythms in sensory processes: A review of fluctuations in vision, olfaction, audition, taste, and touch. Psychological Bulletin, 93, 539-548.

Piaget, J. (1969). Mechanisms of perception (G. N. Seagrim, Trans.). New York: Basic Books. (Original work published 1961)

Scher, D., Pionk, M., \& Purcell, D. G. (1981). Visual sensitivity fluctuations during the menstrual cycle under dark and light adaptation. Bulletin of the Psychonomic Society, 18, 159-160.

Shaffer, G. S., \& PAYNe, R. B. (1982). Contralateral transfer of reactive inhibition as a function of sex and interpolated rest. Perceptual \& Motor Skills, 54, 979-985.

(Manuscript received for publication August 11, 1987.) 